\title{
Shrink pattern of breast cancer after neoadjuvant chemotherapy and its correlation with clinical pathological factors
}

Shushu Wang, Yi Zhang ${ }^{*}$ Xinhua Yang, Linjun Fan, Xiaowei Qi, Qingqiu Chen and Jun Jiang

\begin{abstract}
Background: Breast conservation therapy (BCS) after neoadjuvant chemotherapy (NCT) can improve patients' quality of life. Currently used intraoperative examination for negative margins may not be sufficient to detect microresidual foci, which are a risk factor for local recurrence. This study was conducted to investigate the shrinking pattern of breast cancer and residual tumors as a risk factor for BCS after NCT.

Methods: Ninety women with stage II or III invasive ductal carcinoma who achieved partial response after NCT with paclitaxel and epirubicin were enrolled. All patients had undergone modified radical mastectomy. One-half of the surgical specimens were subjected to subserial sectioning. Pathological changes of tumor bed and pericancerous tissues were examined with an optical microscope. The levels of estrogen receptors, progesterone receptors and HER2 were analyzed by immnohistochemical staining.

Results: The residual tumors were classified into three types according to their microscopic morphology: solitary lesion, multifocal and patchlike lesions, and main residual tumor with satellite lesions. Type I residual tumors were found in 55 patients (61\%), type II in 30 patients (33\%) and type III in 5 patients (6\%). Types II and III were often associated with larger primary tumors. The types of residual tumors were not correlated with the status of hormone receptors or HER2.

Conclusion: Three types of residual tumors were observed after NCT. The solitary residual tumor is most common, but main residual tumors with satellite lesions are most likely to cause local recurrence after BCS. Subserial sectioning would improve the identification of microfoci and patient survival after BCS.
\end{abstract}

Keywords: Breast cancer, Breast conservation surgery, Neoadjuvant chemotherapy, Residual tumor, Subserial section

\section{Background}

Breast conservation therapy (BCS) can improve patients' quality of life. Large-scale, multicenter, randomized controlled trials have shown no significant difference in recurrence-free survival and overall survival (OS) between patients who have undergone BCS and those who have had radical mastectomy [1] in early breast cancer. BCS entails a local recurrence rate of $10 \%$ in early breast cancer, so there is an emphasis on ways of decreasing local recurrence rate after BCS.

\footnotetext{
* Correspondence: jcbd@medmail.com.cn

Breast Disease Center, Southwest Hospital, Third Military Medical University, 29 Gaotanyan Street, Chongging 400038, China
}

Neoadjuvant chemotherapy (NCT) can make more patients suitable for BCS by downstaging primary tumors $[2,3]$. However, NCT-downstaged tumors have a higher local recurrence rate than the primary tumors suitable for BCS, which may be a result of incomplete removal of cancer tissues [4].

Intraoperative frozen section examination is used to ensure negative margins. Some patients still develop local recurrence after BCS, however, despite negative margins reached. Recurrence is often near the site of the original tumor. Concentric or patchlike residual tumors remain at cancer sites after NCT. The patchlike residual cancer is one of the major factors underlying local recurrence after BCS [5]. Conventional pathological methods
C Biomed Central

(c) 2013 Wang et al.; licensee BioMed Central Ltd. This is an Open Access article distributed under the terms of the Creative Commons Attribution License (http://creativecommons.org/licenses/by/2.0), which permits unrestricted use, distribution, and reproduction in any medium, provided the original work is properly cited. 
cannot reveal the general state of a tumor shrinking pattern and may miss microfoci, owing to specimen limitation. In this study, subserial sectioning was used to check the shrinking pattern and identify residual tumors after NCT. Our study should provide information that will help reduce local recurrence and enhance the survival of patients who have undergone BCS.

\section{Methods}

\section{Patients and general protocols}

All patients were enrolled and treated at the Breast Disease Center of the Southwest Hospital between 1 September 2007 and 1 October 2009. We enrolled 90 patients with stage II or III breast cancer. All patients were females from age 28 to about $70 \mathrm{yr}$ (mean: 48.99 yr) (Table 1). Cancers were confirmed by core needle biopsy before NCT. Before chemotherapy or surgery, color Doppler ultrasound and X-ray examinations were performed to evaluate tumor size. Patients were given a paclitaxel and epirubicin (TE) regimen (paclitaxel $175 \mathrm{mg} / \mathrm{m}^{2}$, epirubicin $\mathrm{HCl} 80 \mathrm{mg} / \mathrm{m}^{2}$ iv, $21 \mathrm{~d}$ per round) for three to eight rounds. Routine blood, liver, kidney and heart function was examined before each round of treatment. Prior to surgery, ultrasound and Xray examinations were performed again, the largest tumor diameter after chemotherapy was measured and lesion calcification and type or any small satellite lesions near the main mass were observed. All protocols were approved by the Ethics Committee of Third Military Medical University, and every patient signed the informed consent form.

\section{Inclusion criteria}

1. Stage II (diameter of 2 to $5 \mathrm{~cm})$ or stage III $(>5 \mathrm{~cm}$ without involvement of skin or chest wall) invasive ductal carcinoma confirmed by core needle biopsy.

Table 1 Patients' clinical characteristics

\begin{tabular}{ccc}
\hline Characteristics & No. of patients & $\%$ \\
\hline Age $(y r)$ & 18 & \\
$\leq 40$ & 65 & 20.0 \\
41 to 60 & 7 & 72.2 \\
$>60$ & & 7.8 \\
Chemotherapy (cycle) & 80 & 88.9 \\
$\leq 4$ & 10 & 11.1 \\
$>4$ & & \\
T staging & 14 & 15.6 \\
T1 & 63 & 70.0 \\
T2 & 13 & 14.4 \\
T3 & &
\end{tabular}

2. No involvement of lymph node determined by axillary dissection.

3. Color ultrasound and X-ray examinations showing that the primary tumor was a solitary lesion.

4. Response Evaluation Criteria in Solid Tumors (RECIST) [6] was used and partial response reached.

5. No chemotherapy, radiotherapy, hormonal therapy or any resection biopsy before surgery.

6. Not fit for BCS or patient had personally asked for modified radical mastectomy.

\section{Exclusion criteria}

1. Patients diagnosed with primary cancer with multiple lesions.

2. Partial response not achieved after chemotherapy.

3. Clinically complete relief obtained after chemotherapy.

4. Patients undergoing BCS.

5. Tumor invasion of chest and skin.

6. Patients with stage I or IV breast cancer.

7. Patients who had undergone chemotherapy, radiotherapy, endocrine therapy and resection biopsy previously.

\section{Subserial section of whole breast}

Subserial sections of the whole breast were prepared from the specimens. The sample was put on a board with the cutting surface facing downward, surrounding fat tissue and aponeurosis were cut off and the spindle-shaped skin border was sutured with silk and fixed to the board to prevent skin retraction. The specimen was stored in a $-20^{\circ} \mathrm{C}$ ice box for $24 \mathrm{~h}$, then parallel lines were drawn every $5 \mathrm{~mm}$ from the cutting edge to the margin of the half breast sample. Using these lines, 5 -mm tissue pieces were cut, retrimmed to remove surrounding fat tissue and numbered sequentially. The tissue pieces were fixed in $10 \%$ formalin for $48 \mathrm{~h}$, dehydrated with acetone and anhydrous alcohol for 12 and $24 \mathrm{~h}$ separately, soaked in chloroform and xylene separately for $24 \mathrm{~h}$ each, wax-dipped for 24 hours (melting point, $56^{\circ} \mathrm{C}$ to $58^{\circ} \mathrm{C}$ ) and embedded in paraffin. Eight- to twelve-micrometer sections were cut using a Leica RM2016 slicer (Leica Biosystems, Nussloch, Germany) and stained with hematoxylin and eosin.

Histological evaluation was done according to the method of Miller and Payne [7]. The shape, distribution and size of the residual lesion, as well as hyperplasia of fibrous tissue, lymphocyte infiltration and change of pericancerous tissue, were recorded.

\section{Detection of estrogen receptors, progesterone receptors and HER2}

The levels of estrogen receptors (ERs), progesterone receptors (PRs) and HER2 in preoperative core needle 
biopsy tissues and final surgical specimens were examined by immunohistochemical staining. ER and PR levels were determined by Allred score [8], which is based on the percentage of tumor cells (PS), intensity of the staining (IS) and total score (TS = PS + IS). PS represented the estimated proportion of tumor cells staining positive as follows: 0 (none), 1 (tumor cells staining positive $<1 \%$ ), 2 (tumor cells staining positive between $1 \%$ and 10\%), 3 (tumor cells staining positive between 10\% and 33\%), 4 (proportion of tumor cells staining positive between $33 \%$ and $67 \%$ ) and 5 (proportion of tumor cells staining positive $>67 \%$ ). IS represented the average intensity of the positive cells as follows: 0 (none), 1 (weak), 2 (intermediate) and 3 (strong). PS and IS were then added to obtain TS as follows: negative ( 0 to about 2$), 1+$ ( 3 to about 4$), 2+$ (5 to about 6 ), and $3+$ (7 to about 8 ).

A herceptin test [9] was used to detect HER2 levels. It was scored according to cell membrane staining, indicated as $3+$ (strong, complete membrane staining in $>10 \%$ of tumor cells), $2+$ (weak to moderate, complete membrane staining in $>10 \%$ of the tumor cells), $1+$ (faint membrane staining involving only a portion of the membrane in $>10 \%$ of tumor cells) or 0 (no staining or faint staining in $<10 \%$ of the tumor cells).

\section{Statistical analysis}

SPSS version 13.0 software (SPSS, Inc, Chicago, IL, USA) was used for data analysis. A paired test was applied for matched data and $X^{2}$ and nonparametric tests were done for other data, with $P<0.05$ regarded as significant.

\section{Results}

Light microscopic observation of pathological changes in tumor bed and pericancerous tissues after NCT

There were fibroplasias and lymphocyte infiltration to different degrees near the degenerated cancer site and small blood vessels (Figure 1). Histological classification after chemotherapy showed grade I in 4 cases, grade II in 26, grade III in 51 and grade IV in 10. Twenty-four cases with severe lymphocyte infiltration and sixty-six with light to moderate lymphocyte infiltration were found. Five cases had an intraductal carcinoma component.

\section{Morphological typing of residual breast cancer after NCT}

There were three microscopic morphological types of residual breast cancer after NCT. Type I comprised solitary lesions in the fibrotic tumor bed with extensive lymphocyte infiltration near the cancer lesion (Figure 1A). Type II involved fibrotic changes in the tumor bed, which divided the residual cancer structure into several lesions with irregular shapes and patchlike shapes of different sizes (Figure 1B). Type III had a main cancer lesion and one or two small satellite lesions at least $1.0 \mathrm{~cm}$ away from it $(10 \mathrm{~mm}$ to about $25 \mathrm{~mm}$ ) visible in the fibrotic cancer bed with or without scattered cancer cell structures around the cancer lesion (Figures $1 \mathrm{C} 1$ and $1 \mathrm{C} 2$ ). Among the 90 patients, type I residual cancer was found in 55 (61\%), type II in $30(33 \%)$ and type III in $5(6 \%)$. Among the patients with type III residual cancer, four had one satellite lesion and one had two satellite lesions.

\section{Mammary X-ray features of calcification and satellite lesions of breast cancer after chemotherapy}

Mammary X-ray examination after NCT showed calcification in nine cases. Three had clusterlike granules around the tumor, one had linelike calcification distributed along the breast ducts and five showed large calcification particles located within the tumor. No satellite lesions were found by X-ray examination.

\section{Maximum tumor diameters measured by ultrasonography} and pathological examination

Tumor size was measured after NCT using color Doppler ultrasound. The maximum diameter was 8 to $49 \mathrm{~mm}$ (mean, $19.2 \mathrm{~mm}$ ). Pathological observation of postoperative subserial sections of whole breasts showed that the maximum tumor diameter was 5 to $51 \mathrm{~mm}$ (mean, $21.7 \mathrm{~mm}$ ). The paired $t$-test showed no statistical difference in stage I breast cancer $(P>0.05)$, but significant differences in stages II and III breast cancer $(P<$ 0.05 ) between the two examination methods were found.

\section{Relationship between types of residual tumor, age and menstrual status}

The proportions of three age groups $(\leq 40,41$ to 60 and $>60$ years of age) were $21.8 \%, 70.9 \%$ and $7.3 \%$, respectively, for type I residual tumors; $13.3 \%, 80.0 \%$ and $6.7 \%$, respectively, for type II residual tumors; and 20.0\%, $60.0 \%$ and $20.0 \%$, respectively, for type III residual tumors. There was no statistically significant difference among the three types $(P>0.05)$. The proportions of postmenopausal patients in the three types were $20.0 \%$, $56.7 \%$ and $20.0 \%$, respectively, which had no statistical significance (Table 2).

\section{Relationship between types of residual tumor and primary tumor T stage}

For type I residual tumors, the proportions of T1, T2 and $\mathrm{T} 3$ primary tumors were $20.0 \%, 76.4 \%$ and $3.6 \%$, respectively. There were $10.0 \%, 56.7 \%$ and $33.3 \%$ of type II residual tumors resulting from $\mathrm{T} 1, \mathrm{~T} 2$, and $\mathrm{T} 3$ primary tumors, respectively, whereas $20.0 \%, 60.0 \%$ and $20.0 \%$ of type III residual tumors came from T1, T2, and T3 primary tumors after NCT. The primary tumor stage distribution among the three types of residual cancer was 

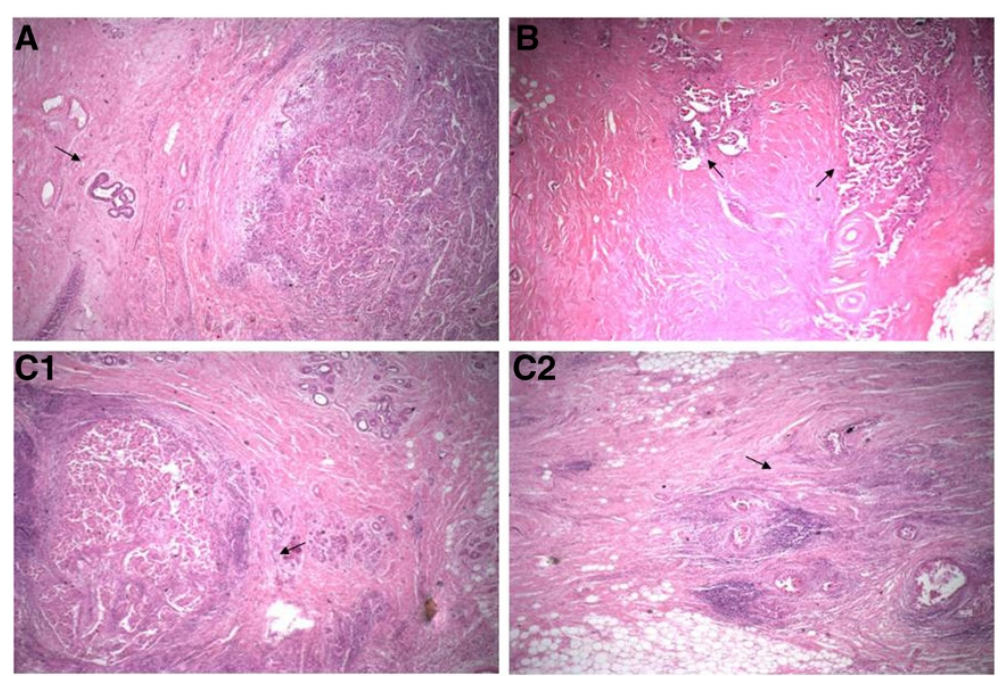

Figure 1 Three types of residual cancers. (A) Type I residual cancer. Solitary residual breast cancer can be seen (arrow). There is a large quantity of lymphocyte infiltration in the margin of the cancer lesion. There are no residual cancer cells, cancer cell clumps or satellite lesions near the cancer lesion. (B) Type II residual cancer. Two residual cancer lesions can be seen (arrows), and there is fibrotic change between the lesions. (C) Type III residual tumor. Solitary residual lesion (C1, arrow) and satellite lesion $1.5 \mathrm{~cm}$ away from the main lesion (C2, arrow) can be seen (hematoxylin and eosin stain; original magnification, $\times 40$ ).

statistically different $(P<0.05)$. Type II and type III residual tumors had higher occurrences in patients with larger primary tumors.

\section{Relationship between types of residual tumor and levels of ER, PR and HER2}

The positive rates of ER, PR and HER2 before and after NCT were not statistically different among patients with three types of residual tumors $(P>0.05)$ (Table 3$)$. The changes in expression levels of ER, PR and HER2 among three types of residual cancers also were not statistically different $(P>0.05)$ (Table 4$)$.

\section{Discussion}

It has been shown that patients who have undergone modified radical mastectomy have a long-term survival rate similar to that of those who have undergone radical mastectomy [10-13]. NCT can make $23 \%$ of primary breast cancers that are otherwise unfit for BCS manageable with BCS through downstaging [4]. Wolmark et al.
[3] reported a local recurrence rate of $10.7 \%$ in postchemotherapy patients treated with $\mathrm{BCS}, 7.6 \%$ in patients with primary tumors fit for BCS and $15.9 \%$ in patients with primary breast cancer unfit for BCS through downstaging by chemotherapy. The local recurrences were found mostly around the site of operation.

MRI observations of tumor morphological changes after NCT have been classified by contraction patterns into concentric contraction and patchlike contraction [2]. About $70 \%$ of residual tumors were previously found to be solitary and 30\% were patchlike [5]. A negative surgical margin of BCS is closely associated with local recurrence-free and metastasis-free survival $[3,4]$. It was difficult to ensure negative surgical margins in BCS patients who had multifocal residual tumors, which was one of the factors that caused local recurrence [14,15]. However, some patients with solitary residual tumors that had negative surgical margins still developed local recurrence. There might be microfoci that are missed by conventional histological sections due to sample

Table 2 Relationship between types of residual tumor with age and menstrual status

\begin{tabular}{|c|c|c|c|c|c|c|}
\hline \multirow[t]{2}{*}{ Types } & \multirow[t]{2}{*}{$n$} & \multicolumn{3}{|c|}{ Age (yr) (\%) } & \multicolumn{2}{|c|}{ Menstrual status (\%) } \\
\hline & & $\leq \mathbf{4 0}$ & 41 to 60 & $>60$ & Postmenopausal & Premenopausal \\
\hline Type I & 55 & $12(21.8)$ & $39(70.9)$ & $4(7.3)$ & $22(20.0)$ & $33(80.0)$ \\
\hline Type II & 30 & $4(13.3)$ & $24(80.0)$ & $2(6.7)$ & $17(56.7)$ & $13(43.3)$ \\
\hline Type III & 5 & $1(20.0)$ & $3(60.0)$ & $1(20.0)$ & $1(20.0)$ & $4(80.0)$ \\
\hline$x^{2}$ & & & & & \multicolumn{2}{|c|}{3.465} \\
\hline$P$ value ${ }^{\mathrm{a}}$ & & & 0.393 & & \multicolumn{2}{|c|}{0.177} \\
\hline
\end{tabular}

${ }^{a}$ Kruskal-Wallis test and $x^{2}$ test. 
Table 3 Relationship between types of residual tumor and expression of ER ${ }^{a}$, PR $^{a}$ and HER2 ${ }^{b}$

\begin{tabular}{|c|c|c|c|c|c|c|c|c|}
\hline \multirow[t]{2}{*}{ Types } & \multicolumn{4}{|c|}{ No. of ER (\%) prior to NCT } & \multicolumn{4}{|c|}{ No. of ER (\%) after NCT } \\
\hline & - & + to about +++ & $x^{2}$ & $P$ value & - & + to about +++ & $x^{2}$ & $P$ value \\
\hline Type I & $21(38.2)$ & $34(61.8)$ & 2.032 & 0.362 & $22(40.0)$ & $33(60.0)$ & 0.378 & 0.828 \\
\hline Type II & $7(23.3)$ & $23(76.7)$ & & & $10(33.3)$ & $20(66.7)$ & & \\
\hline \multirow[t]{3}{*}{ Type III } & $2(40.0)$ & $3(60.0)$ & & & $2(40.0)$ & $3(60.0)$ & & \\
\hline & \multicolumn{4}{|c|}{ No. of PR (\%) prior to NCT } & \multicolumn{4}{|c|}{ No. of PR (\%) after NCT } \\
\hline & - & + to about +++ & $x^{2}$ & $P$ value & - & + to about +++ & $x^{2}$ & $P$ value \\
\hline Type I & $13(23.6)$ & $42(76.4)$ & 0.499 & 0.779 & $13(23.6)$ & $42(76.4)$ & 0.153 & 0.926 \\
\hline Type II & $9(30.0)$ & $21(70.0)$ & & & $8(26.7)$ & $22(73.3)$ & & \\
\hline \multirow[t]{3}{*}{ Type III } & $1(20.0)$ & $4(80.0)$ & & & $1(20.0)$ & $4(80.0)$ & & \\
\hline & \multicolumn{4}{|c|}{ No. of HER2 (\%) before NCT } & \multicolumn{4}{|c|}{ No. of HER2 (\%) after NCT } \\
\hline & - to about + & ++ to about ++++ & $x^{2}$ & $P$ value & - to about + & ++ to about +++ & $x^{2}$ & $P$ value \\
\hline Type I & $32(58.2)$ & $23(41.8)$ & 0.743 & 0.154 & 39 (70.9) & $16(29.1)$ & 1.142 & 0.565 \\
\hline Type II & $24(80.0)$ & $6(20.0)$ & & & $24(80.0)$ & $6(20.0)$ & & \\
\hline Type III & $4(80.0)$ & $1(20.0)$ & & & $4(80.0)$ & $1(20.0)$ & & \\
\hline
\end{tabular}

${ }^{a} x^{2}$ test. ${ }^{b}$ Kruskal-Wallis test.

selection but could be revealed by subserial sectioning of the whole breast.

Successive breast subsections were mostly used to study multifocal primary breast cancer lesions, the scattering mode of breast cancer, Paget's disease of the breast and diagnosis of occult breast cancer [16-18]. We used subserial sectioning of the whole breast to study the morphology of residual breast cancer and the changes in the tumor bed. The morphology of residual tumors after NCT observed by pathological examination was similar to the shrink patterns examined by imaging. Besides the two previously reported types of residual breast cancer after NCT, for example, solitary, multifocal and patchlike lesions, a third type of residual tumor which has one main cancer lesion and one or two small satellite lesions at least $1.0 \mathrm{~cm}$ away from it was also observed. As the satellite lesions are located

Table 4 Relationship between types of residual tumors and the expression changes

\begin{tabular}{cccccc}
\hline Receptor & Change & \multicolumn{4}{c}{ Type of residual tumor } \\
\cline { 3 - 6 } & & Type I & Type II & Type III & $\boldsymbol{P}$ value $^{\mathbf{a}}$ \\
\hline ER & Increase & 2 & 2 & 0 & 0.839 \\
& No change & 41 & 20 & 4 & \\
& Decrease & 12 & 8 & 1 & \\
PR & Increase & 6 & 3 & 0 & 0.891 \\
& No change & 36 & 20 & 4 & \\
& Decrease & 13 & 7 & 1 & \\
\multirow{2}{*}{ HER2 } & Increase & 6 & 2 & 1 & 0.545 \\
& No change & 36 & 22 & 4 & \\
& Decrease & 13 & 6 & 0 & \\
\hline
\end{tabular}

Jonckheere-Terpstra test. away from the main residual tumor, the surgical margin may appear negative when examined by intraoperative frozen biopsy, but remaining satellite lesions could cause local recurrence.

The pattern of residual tumor is thought to relate to primary tumor size, and multifocal patchlike lesions more frequently result from larger primary tumors [19]. We have shown that the larger the primary tumors, the more types II and III residual tumors occurred, which may be due to vascular distribution and avascular necrosis. Age and menstrual status had no bearing on the pattern of residual tumors.

Chagpar et al. suggested that ultrasound and X-ray examinations cannot identify residual tumors and chemotherapy-related fibrotic changes, as X-ray underestimates the volume of larger tumors and ultrasound underestimates all breast lumps [20]. We detected nine cases of calcification with X-ray examination after NCT. Pathological examination showed that five of the nine cases of calcification had an intraductal cancer component, and four showed small blood vessel changes within the cancer or necrosis around blood vessels. For type I residual tumors, there was no difference in tumor size measured by ultrasound and pathological examination. However, there was a significant size difference between measurement by ultrasound and pathological examination of types II and III residual tumors. Ultrasound may not have enough sensitivity to penetrate the larger size of types II and III tumors.

It has been shown that the efficacy of NCT may be influenced by ER, PR and HER2 status [21-23]. The response to chemotherapy was different with regard to the types of cancer and drugs involved. Patients with triplenegative breast cancers had better responses to NCT 
and better long-term survival [22]. We looked at the possible correlation between ER, PR and HER2 status with the types of residual tumors after NCT. The levels of or the change of levels of ER, PR and HER2 were similar among cancers with different types of residual tumors after NCT, indicating that the impact of type II and type III on local recurrence and survival rate was not related to ER, PR or HER2 status.

\section{Conclusion}

Patients with solitary lesions after chemotherapy were suitable for BCS, whereas the ones with multifocal and patchlike residual lesions required extra caution. BCS negative margins of type III residual tumors obtained from intraoperative frozen biopsies might miss satellite lesions and lead to local recurrence.

\section{Abbreviations \\ BCS: Breast conservation therapy; ER: Estrogen receptor; HER2: Human epidermal growth factor receptor 2; OS: Overall survival; PR: Progesterone receptor; RECIST: Response evaluation criteria in solid tumors; TE: Taxol and epirubicin.}

\section{Competing interests}

The authors declare that they have no competing interests.

\section{Authors' contributions}

$S W, X Y, L E, X Q$ and $Q C$ carried out data acquisition. $Y Z$ and JJ conceived the project and designed the study. SW, YZ and JJ drafted the manuscript. $\mathrm{XQ}$ and $\mathrm{J}$ carried out statistical analyses. All authors read and approved the final manuscript.

\section{Acknowledgements}

This work was funded by the Clinical Innovation Fund of Southwest Hospital, Third Military Medical University.

Received: 22 March 2013 Accepted: 8 July 2013

Published: 24 July 2013

\section{References}

1. Poggi MM, Danforth DN, Sciuto LC, Smith SL, Steinberg SM, Liewehr DJ, Menard C, Lippman ME, Lichter AS, Altemus RM: Eighteen-year results in the treatment of early breast carcinoma with mastectomy versus breast conservation therapy: the national cancer institute randomized trial. Cancer 2003, 98:697-702.

2. Fisher B, Brown A, Mamounas E, Wieand S, Robidoux A, Margolese RG, Cruz AB Jr, Fisher ER, Wickerham DL, Wolmark N, DeCillis A, Hoehn JL, Lees AW, Dimitrov NV: Effect of preoperative chemotherapy on local-regional disease in women with operable breast carcinoma: findings from national surgical adjuvant breast and bowel project B-18. J Clin Oncol 1997, 15:2483-2493.

3. Wolmark N, Wang J, Mamounas E, Bryant J, Fisher B: Preoperative chemotherapy in patients with operable breast cancer: nine-year results from the national surgical adjuvant breast and bowel project B-18. J Natl Cancer Inst Monogr 2001, 30:96-102.

4. van der Hage JA, van de Velde CJ, Julien JP, Tubiana-Hulin M, Vandervelden C, Duchateau L: Preoperative chemotherapy in primary operable breast cancer: results from the European organization for research and treatment of cancer Trial 10902. J Clin Oncol 2001, 19:4224-4237.

5. Chen AM, Meric-Bernstam F, Hunt KK, Thames HD, Oswald MJ, Outlaw ED, Strom EA, McNeese MD, Kuerer HM, Ross MI, Singletary SE, Ames FC, Feig BW, Sahin AA, Perkins GH, Schechter NR, Hortobagyi GN, Buchholz TA: Breast conservation after neoadjuvant chemotherapy: the MD Anderson cancer center experience. J Clin Oncol 2004, 22:2303-2312.

6. Eisenhauer EA, Therasse P, Bogaerts J, Schwartz LH, Sargent D, Ford R, Dancey J, Arbuck S, Gwyther S, Mooney M, Rubinstein L, Shankar L, Dodd L,
Kaplan R, Lacombe D, Verweij J: New response evaluation criteria in solid tumors: revised RECIST guideline (version 1.1). Eur J Cancer 2009, 45:228-247.

7. Ogston KN, Miller ID, Payne S, Hutcheon AW, Sarkar TK, Smith I, Schofield A, Heys SD: A new histological grading system to assess response of breast cancers to primary chemotherapy: prognostic significance and survival. Breast 2003, 12:320-327.

8. Harvey JM, Clark GM, Osborne CK, Allred DC: Estrogen receptor status by immunohistochemistry is superior to the ligand-binding assay for predicting response to adjuvant endocrine therapy in breast cancer. J Clin Oncol 1999, 17:1474-1481.

9. Group of guideline of HER2 detection in breast cancer: Guideline of HER2 detection in breast cancer [in Chinese]. Zhonghua Bing Li Xue Za Zhi 2006, 35:631-633.

10. Bodian CA, Perzin KH, Lattes R: Lobular neoplasia: long-term risk of breast cancer and relation to other factors. Cancer 1996, 78:1024-1034.

11. Fisher ER, Costantino J, Fisher B, Palekar AS, Paik SM, Suarez CM, Wolmark N: Pathologic findings from the national surgical adjuvant breast project (NSABP) protocol B-17: five-year observations concerning lobular carcinoma in situ. Cancer 1996, 78:1403-1416.

12. Pendas S, Dauway E, Giuliano R, Ku N, Cox CE, Reintgen DS: Sentinel node biopsy in ductal carcinoma in situ patients. Ann Surg Oncol 2000, 7:15-20.

13. Kestin LL, Goldstein NS, Martinez AA, Rebner M, Balasubramaniam M, Frazier RC, Register JT, Pettinga J, Vicini FA: Mammographically detected ductal carcinoma in situ treated with conservative surgery with or without radiation therapy: patterns of failure and 10-year results. Ann Surg 2000, 231:235-245.

14. Buchholz TA, Hunt KK, Whitman GJ, Sahin AA, Hortobagyi GN: Neoadjuvant chemotherapy for breast carcinoma: multidisciplinary considerations of benefits and risks. Cancer 2003, 98:1150-1160.

15. Peintinger F, Kuerer HM, McGuire SE, Bassett R, Pusztai L, Symmans WF: Residual specimen cellularity after neoadjuvant chemotherapy for breast cancer. Br J Surg 2008, 95:433-437.

16. Zhong L, He G, Chen Q, Fan L, Jiang J, Zhang Y: Exploration on the technique and application of whole breast serial subsection [in Chinese]. Chin J Breast Dis 2009, 5:564-567.

17. Zhang W, Ma R, Zhang Y, Fu J, Yao Y, Xu J: Clinicopathological study of the nipple-areolar complex involvement in breast cancer [in Chinese]. Chin J Pract Surg 2003, 10:35-36.

18. Zhang Y, Zhou Y, Guo M, Guo D, Feng C, Jiang J: Infiltrating characteristics of breast cancer after neoadjuvant chemotherapy: whole breast serial subsection observation [in Chinese]. Di 3 Jun Yi Da Xue Xue Bao 2007, 21:2083-2085.

19. Veronesi U, Bonadonna G, Zurrida S, Galimberti V, Greco M, Brambilla C, Luini A, Andreola S, Rilke F, Raselli R: Conservation surgery after primary chemotherapy in large carcinomas of the breast. Ann Surg 1995, 222:612-618.

20. Chagpar AB, Middleton LP, Sahin AA, Dempsey P, Buzdar AU, Mirza AN, Ames FC, Babiera GV, Feig BW, Hunt KK, Kuerer HM, Meric-Bernstam F, Ross MI, Singletary SE: Accuracy of physical examination, ultrasonography, and mammography in predicting residual pathologic tumor size in patients treated with neoadjuvant chemotherapy. Ann Surg 2006, 243:257-264.

21. Kasami M, Uematsu T, Honda M, Yabuzaki T, Sanuki J, Uchida Y, Sugimura H: Comparison of estrogen receptor, progesterone receptor and Her-2 status in breast cancer pre- and post-neoadjuvant chemotherapy. Breast 2008, 17:523-527.

22. Hannemann J, Oosterkamp HM, Bosch CA, Velds A, Wessels LF, Loo C, Rutgers EJ, Rodenhuis S, van de Vijver MJ: Changes in gene expression associated with response to neoadjuvant chemotherapy in breast cancer. J Clin Oncol 2005, 23:3331-3342.

23. Learn PA, Yeh IT, McNutt M, Chisholm GB, Pollock BH, Rousseau DL Jr, Sharkey FE, Cruz AB, Kahlenberg MS: HER-2/neu expression as a predictor of response to neoadjuvant docetaxel in patients with operable breast carcinoma. Cancer 2005, 103:2252-2260.

doi:10.1186/1477-7819-11-166

Cite this article as: Wang et al:: Shrink pattern of breast cancer after neoadjuvant chemotherapy and its correlation with clinical pathological factors. World Journal of Surgical Oncology 2013 11:166. 